\title{
Implementation of behavioral systems
}

\author{
Diego Napp and Paula Rocha
}

Lecture Notes in Control and Information Sciences, Vol. 4, pp. 151-168
DOI: 10.1007/978-3-319-21003-2_8

\begin{abstract}
In this paper, we study control by interconnection of a given linear differential system (the plant behavior) with a suitable controller. The problem formulations and their solutions are completely representation free, and specified only in terms of the system dynamics. A controller is a system that constrains the plant behavior through a certain set of variables. In this context, there are two main situations to be considered: either all the system variables are available for control, i.e., are control variables (full control) or only some of the variables are control variables (partial control). For systems evolving over a time domain (1D) the problems of implementability by partial (regular) interconnection are well-understood. In this paper we study why similar results are not valid in the multidimensional (nD) case. Finally, we study two important classes of controllers, namely, canonical controllers and regular controllers.
\end{abstract}

\section{Introduction}

It is a pleasure to contribute an article in honor of Harry L. Trentelman on the occasion of his 60-th birthday. The first author had the privilege of being one of his $\mathrm{PhD}$ students and of developing a fruitful research collaboration with him over the last decade. Although she never directly collaborated with Harry, the second author has always appreciated his work, by which she was inspired in several occasions.

Diego Napp

CIDMA - Center for Research and Development in Mathematics and Applications, Department of Mathematics, University of Aveiro, Campus Universitario de Santiago, 3810-193 Aveiro, Portugal, e-mail: diego@ua.pt,

Paula Rocha

SYSTEC - Research Center for Systems and Technologies, Department of Electrical and Computer Engineering, Faculty of Engineering, University of Porto, Portugal, e-mail: mprocha@fe.up.pt 
As the topic of our article, we have chosen an issue which is at the core of systems and control theory, namely control and, in particular, the implementation of systems in the behavioral framework. This topic goes back to the seminal contribution of $\mathrm{J}$. C. Willems in [17] where the fundamental ideas of the problem were established. However, it was Harry who thoroughly investigated this issue and provided many fundamental results in this area. It is our intention to make this article an appropriate tribute to his wide ranging scientific interests and to the influence that his work had in the field of behavioral approach to systems and control theory. For this purpose, we have gathered in this paper our results that are more connected with Harry's own research, together with some new results and insights. In order to keep the paper self-contained and to give a better idea of the kind of reasoning involved, we have included the proofs of most of those results.

A behavior, denoted by $\mathfrak{B}$, is a set of trajectories that obey certain laws described by a mathematical model. In this context, control is viewed as the ability to impose adequate additional restrictions to the variables of the behavior in order to obtain a desired overall functioning pattern. Hence, the behavioral approach proposes a new perspective to control which is based on interconnection of systems, and where no a priori input/output partition is considered [17]. The act of controlling a system is simply viewed as intersecting its behavior with a controller behavior in order to achieve a desired behavior. Thus, a general control (implementation) problem can be stated as follows: Given a plant behavior $\mathfrak{B}$ and a control objective corresponding to a desired behavior that we want to implement $\mathcal{K}$, find a controller behavior $\mathfrak{B}^{c}$, within a certain controller class, such that the behavior resulting from the interconnection of $\mathfrak{B}$ and $\mathfrak{B}^{c}, \mathfrak{B} \cap \mathfrak{B}^{c}$, coincides with $\mathcal{K}$.

Most of the literature on behavioral control is concerned with the situation in which all variables of $\mathfrak{B}$ are available for control, i.e., it is allowed to impose extra restrictions on all the variables of $\mathfrak{B}$. We refer to this situation as full control or full interconnection $[10,11,17]$. Another important case considered in the literature is when the system variables are divided into two sets: the variables that we are interested to control (called to-be-controlled variables) and the variables on which we are allowed to enforce restrictions (called control variables). This situation is known as partial control or partial interconnection $[1,4,12,15,18]$. In this more involved situation, although we can not act directly upon the to-be-controlled variables, we can nevertheless influence their dynamics by imposing restrictions on the control variables.

Of particular interest is the kind of interconnection that is called regular interconnection. In such interconnection, the restrictions imposed on the plant by the controller are independent of the restrictions already present in the plant. These type of interconnections are closely related to the notion of feedback control in the classical state-space systems since only system inputs are restricted, as in a feedback loop $[14,17]$.

The first results on implementability of full control problems were obtained in $[18,20]$ for linear systems evolving over a time domain (1D behaviors) and in [16] 
for a very general class of systems. Later, results for 1D behaviors were generalized to regular partial interconnections in [1] (see also [2, 12, 19]). In the context of multidimensional systems ( $\mathrm{nD}$ behaviors) full regular interconnections were first investigated in $[14,24]$ and results on the partial interconnection counterpart were first presetned in $[13,15]$. The case of $\mathrm{nD}$ behaviors constituted by compactly supported functions was investigated in [8].

The problem of implementability by regular interconnections is well understood and fully characterized for 1D behaviors in both contexts of full and partial control, see for instance $[1,10,11,18]$. In fact, $\mathcal{K} \subset \mathfrak{B}$ is implementable by regular full interconnection if and only if $\mathfrak{B}=\mathfrak{B}^{c}+\mathcal{K}$ where $\mathfrak{B}^{c}$ is the controllable part of $\mathfrak{B}$. Moreover, in [1] the solvability of a $1 \mathrm{D}$ partial control problem was related to the solvability of a suitable associated full control problem involving only the to-be-controlled variables and also in terms of the controllable and autonomous parts of the behavior. The situation in the $\mathrm{nD}$ case is somewhat more involved, and a direct characterization in terms of implementation of the to-be-controlled variables seems to be impossible. In this paper our aim is to reinvestigate the problem of implementability by full and partial regular interconnections of $\mathrm{nD}$ behaviors. More concretely, we study the role of the so-called hidden behavior and also of the controllable-autonomous decomposition.

This paper is organized as follows: we begin by introducing some necessary background from the field of $n \mathrm{D}$ behaviors, centering around concepts such as controllability, autonomy, orthogonal module, etc. We conclude this section with a subsection on behaviors with two types of variables. Section 3 is devoted to the study of the problem of implementation by regular interconnection. We first analyze the implementation by full control to conclude the paper by treating the more general case of implementation by partial interconnections.

\section{Preliminaries}

In order to state more precisely the questions to be considered we introduce in this section the necessary material and notation on behavioral theory for $n \mathrm{D}$ systems. The last subsection is concerned with the theory of behaviors with two different types of variables (the to-be-controlled variables and the control variables).

\section{1 nD (kernel) behaviors}

In the behavioral approach to $n \mathrm{D}$ systems, a system or behavior is defined by a triple $(\mathcal{U}, q, \mathfrak{B})$, where $\mathcal{U}$ is the signal space or trajectory universe, $q \in \mathbb{Z}^{+}$is the number of components of the system variable vector, and $\mathfrak{B} \subset \mathcal{U}^{q}$ is the behavior. In this paper, we assume $\mathcal{U}=(\mathbb{C})^{\mathbb{Z}^{n}}$. 
Since the theory of continuous linear time-invariant systems as discussed in [21] is completely analogous to that of the present paper, the same tools and conclusions will apply in the continuous case, where $\mathcal{U}$ is the space of all infinitely often differentiable functions from $\mathbb{R}^{n}$ to $\mathbb{R}$, or all $\mathbb{R}$-valued distributions on $\mathbb{R}^{n}$. For the sake of simplicity we will however focus on the discrete case.

We call $\mathfrak{B}$ a linear difference $n D$ behavior or simply $n D$ behavior if it is the solution set of a system of linear, constant-coefficient partial difference equations, more precisely, if $\mathfrak{B}$ is the subset of $\mathcal{U}^{q}$ given by:

$$
\mathfrak{B}=\operatorname{ker} R\left(\underline{\sigma}, \underline{\sigma}^{-1}\right):=\left\{w \in \mathcal{U}^{q} \mid R\left(\underline{\sigma}, \underline{\sigma}^{-1}\right) w \equiv 0\right\},
$$

$\underline{\sigma}=\left(\sigma_{1}, \ldots, \sigma_{n}\right), \underline{\sigma}^{-1}=\left(\sigma_{1}^{-1}, \ldots, \sigma_{n}^{-1}\right)$, the $\sigma_{i}$ 's are the elementary $n \mathrm{D}$ shift operators (defined by $\sigma_{i} w(\underline{k})=w\left(\underline{k}+e_{i}\right)$, for $\underline{k} \in \mathbb{Z}^{n}$, where $e_{i}$ is the $i$ th element of the canonical basis of $\left.\mathbb{C}^{n}\right)$ and $R\left(\underline{s}, \underline{s}^{-1}\right) \in \mathbb{R}^{p \times q}\left[\underline{s}, \underline{s}^{-1}\right]$ is an $n \mathrm{D}$ Laurent-polynomial matrix known as representation of $\mathfrak{B}$. If no confusion arises, given an $n \mathrm{D}$ Laurentpolynomial matrix $A\left(\underline{\sigma}, \underline{\sigma}^{-1}\right)$, we sometimes write $A$ instead of $A\left(\underline{\sigma}, \underline{\sigma}^{-1}\right)$ and $A\left(\underline{s}, \underline{s}^{-1}\right)$.

Instead of characterizing $\mathfrak{B}$ by means of a representation matrix $R$, it is also possible to characterize it by means of its orthogonal module $\operatorname{Mod}(\mathfrak{B})$, which consists of all the $n \mathrm{D}$ Laurent-polynomial rows $r\left(\underline{s}, \underline{s}^{-1}\right) \in \mathbb{C}^{q}\left[\underline{s}, \underline{s}^{-1}\right]$ such that $\mathfrak{B} \subset \operatorname{ker} r\left(\underline{\sigma}, \underline{\sigma}^{-1}\right)$, and can be shown to coincide with the $\mathbb{C}\left[\underline{s}, \underline{s}^{-1}\right]$-module $\operatorname{RM}(R)$ generated by the rows of $R$, i.e., $\operatorname{Mod}(\mathfrak{B})=\operatorname{RM}\left(R\left(\underline{s}, \underline{s}^{-1}\right)\right)$ [21]. Note that this corresponds to the set of all (linear constant coefficient difference) equations that are satisfied by all the elements (trajectories) of $\mathfrak{B}$.

It turns out that sums, intersections and inclusions of behaviors can be formulated in terms of the corresponding modules.

Theorem 1. [24, p.1074] Let $\mathfrak{B}^{1}$ and $\mathfrak{B}^{2}$ be two behaviors. Then, $\mathfrak{B}^{1}+\mathfrak{B}^{2}$ and $\mathfrak{B}^{1} \cap \mathfrak{B}_{2}$ are also behaviors and

1. $\operatorname{Mod}\left(\mathfrak{B}^{1}+\mathfrak{B}^{2}\right)=\operatorname{Mod}\left(\mathfrak{B}^{1}\right) \cap \operatorname{Mod}\left(\mathfrak{B}^{2}\right)$.

2. $\operatorname{Mod}\left(\mathfrak{B}^{1} \cap \mathfrak{B}^{2}\right)=\operatorname{Mod}\left(\mathfrak{B}^{1}\right)+\operatorname{Mod}\left(\mathfrak{B}^{2}\right)$.

3. $\mathfrak{B}^{1} \subset \mathfrak{B}^{2} \Leftrightarrow \operatorname{Mod}\left(\mathfrak{B}^{2}\right) \subset \operatorname{Mod}\left(\mathfrak{B}^{1}\right)$.

Note that part 3 in Theorem 1 implies that if $\mathfrak{B}^{1}=\operatorname{ker} R_{1} \subset \mathfrak{B}^{2}=\operatorname{ker} R_{2}$, then there exists an L-polynomial matrix $S$ such that $R_{2}=S R_{1}$.

For a full column rank L-polynomial matrix $R \in \mathbb{R}^{p \times q}\left[\underline{s}, \underline{s}^{-1}\right]$ define its Laurent variety (or zeros) as

$$
\mathcal{V}(R)=\left\{\left(\lambda_{1}, \lambda_{2}\right) \in \mathbb{C}^{2} \mid \operatorname{rank}\left(R\left(\lambda_{1}, \lambda_{2}\right)\right)<\operatorname{rank}(R), \lambda_{1} \lambda_{2} \neq 0\right\},
$$

where the first rank is taken over $\mathbb{C}$ and the second one over $\mathbb{R}\left[\underline{s}, \underline{s}^{-1}\right]$. Note that $\mathcal{V}(R)$ is equal to the set of common zeros of the $q \times q$ minors of $R$.

Definition 1. A full column rank L-polynomial matrix $R \in \mathbb{R}^{p \times q}\left[\underline{s}, \underline{s}^{-1}\right]$ is said to be right minor prime (rMP) if $\mathcal{V}(R)$ is finite and right zero prime (rZP) if $\mathcal{V}(R)$ 
is empty. A full row rank L-polynomial matrix $R \in \mathbb{R}^{p \times q}\left[\underline{s}, \underline{s}^{-1}\right]$ is said to be left minor/zero prime ( $\ell \mathrm{MP} / \ell \mathrm{ZP})$ if $R^{T}$ is right minor/zero prime, respectively. An Lpolynomial matrix $L$ is called a minimal left annihilator (MLA) of $R$ if $L R=0$, and for any other L-polynomial matrix $S$ such that $S R=0$ we have that $S=A L$ for some L-polynomial matrix $A$. We define minimal right annihilators in a similar way, with the obvious adaptations.

We next review the notions of controllability and autonomy in the context of the behavioral approach.

Definition 2. A behavior $\mathfrak{B} \subset\left(\mathbb{R}^{q}\right)^{\mathbb{Z}^{n}}$ is said to be controllable if for all $z_{1}, z_{2} \in \mathfrak{B}$ there exists $\delta>0$ such that for all subsets $U_{1}, U_{2} \subset \mathbb{Z}^{n}$ with $d\left(U_{1}, U_{2}\right)>\delta$, there exists a $z \in \mathfrak{B}$ such that $\left.z\right|_{U_{1}}=\left.z_{1}\right|_{U_{1}}$ and $\left.z\right|_{U_{2}}=\left.z_{2}\right|_{U_{2}}$.

In the above definition, $d(\cdot, \cdot)$ denotes the Euclidean metric on $\mathbb{Z}^{n}$ and $\left.z\right|_{U}$, for some $U \subset \mathbb{Z}^{n}$, denotes the trajectory $z$ restricted to the domain $U$.

In contrast with the one dimensional case, $n \mathrm{D}$ behaviors admit a stronger notion of controllability called rectifiability (also known in the literature as strong controllability). Whereas controllable behaviors are the ones that can be represented by an MLA of some L-polynomial matrix or in others words $\mathbb{C}^{q}\left[\underline{s}, \underline{s}^{-1}\right] / \operatorname{Mod}(\mathfrak{B})$ is torsion free, rectifiable behaviors are the ones that can be represented by $\ell \mathrm{ZP}$ matrices, i.e., the $\mathbb{R}\left[\underline{s}, \underline{s}^{-1}\right]$-module $\mathbb{C}^{q}\left[\underline{s}, \underline{s}^{-1}\right] / \operatorname{Mod}\left(\mathfrak{B}^{c}\right)$ is free.

On the other hand, we shall say that a behavior is autonomous if it has no free variables, i.e., no "inputs". It can be shown that $\mathfrak{B}=\operatorname{ker} R$ is autonomous if and only if $R$ has full column rank. In the 1D case, all autonomous behaviors are finite dimensional vector spaces but in the $\mathrm{nD}$ case this is no longer true. Whereas for $1 \mathrm{D}$ systems initial conditions are given in a finite number of points, $\mathrm{nD}$ autonomous systems are generally infinite dimensional. But even in this case the amount of information (initial conditions) necessary to generate the trajectories of an autonomous $\mathrm{nD}$ system may vary. Hence, given an autonomous behavior, a natural question to ask is how much information is necessary in order to fully determine the system trajectories, i.e., how large is the initial condition set. This question has been analyzed in $[5,22]$ by introducing the notion of autonomy degrees for behaviors.

Definition 3. Let $\mathfrak{B}$ be a non-zero autonomous behavior and $R \in \mathbb{R}^{p \times q}\left[\underline{s}, \underline{s}^{-1}\right]$ be an $n \mathrm{D}$ Laurent-polynomial matrix with full column rank such that $\mathfrak{B}=\operatorname{ker} R$. We define autodeg $(\mathfrak{B})=n-\operatorname{dim} \mathcal{V}(R)$ to be the autonomy degree of $\mathfrak{B}$. The autonomy degree of the zero behavior is defined to be $\infty$.

It turns out that the larger the autonomy degree, the smaller is the freedom to assign initial conditions, see [5].

Every $\mathrm{nD}$ behavior $\mathfrak{B}$ can be decomposed into the sum $\mathfrak{B}=\mathfrak{B}^{c}+\mathfrak{B}^{a}$, where $\mathfrak{B}^{c}$ is the controllable part of $\mathfrak{B}$ (defined as the largest controllable sub-behavior of $\mathfrak{B}$ ) and $\mathfrak{B}^{a}$ is a (non-unique) autonomous sub-behavior. This sum can be chosen 
to be direct for 1D behaviors, but this is not always possible for multidimensional behaviors, see [23].

\subsection{Behaviors with two types of variables}

Since in this paper we are interested in considering different types of variables in a behavior (the to-be-controlled variables and the control variables), we introduce the notation $\mathfrak{B}_{(w, c)}$ for a behavior whose variable $z$ is partitioned into two sub-variables $w$ and $c$. Partitioning the corresponding representation matrix as $[R M]$, we can write

$$
\mathfrak{B}_{(w, c)}=\left\{(w, c) \in \mathcal{U}^{\mathrm{w}+\mathrm{c}} \mid R\left(\underline{\sigma}, \underline{\sigma}^{-1}\right) w+M\left(\underline{\sigma}, \underline{\sigma}^{-1}\right) c=0\right\}=\operatorname{ker}[R M] .
$$

In the case one is only interested in analyzing the evolution of one of the subvariables, say, $w$, it is useful to eliminate the other one $(c)$ and consider the projection of the behavior $\mathfrak{B}_{(w, c)}$ into $\mathcal{U}^{\mathrm{w}}$, defined as

$$
\pi_{w}\left(\mathfrak{B}_{(w, c)}\right)=\left\{w \mid \exists c \text { such that }(w, c) \in \mathfrak{B}_{(w, c)}\right\} .
$$

The elimination theorem [9] guarantees that $\pi_{w}\left(\mathfrak{B}_{(w, c)}\right)$ is also a (kernel) behavior, for which a representation can be constructed as follows: take a minimal left annihilator (MLA) $E$ of $M$. Then $\pi_{w}\left(\mathfrak{B}_{(w, c)}\right)=\operatorname{ker}(E R)$, see [9, Cor. 2.38].

On the other hand given a behavior $\mathfrak{B}=\operatorname{ker} R \subset \mathcal{U}^{\text {w }}$ we define the lifting of $\mathfrak{B}$ into $\mathcal{U}^{\mathrm{w}+\mathrm{c}}$ as

$$
\mathfrak{B}_{(w, c)}^{*}:=\left\{(w, c) \in \mathcal{U}^{\mathrm{w}+\mathrm{c}} \mid c \text { is free and } w \in \mathfrak{B}\right\} .
$$

Obviously $\mathfrak{B}_{(w, c)}^{*}=\operatorname{ker}[R 0]$. Analogous definitions can be given if the roles of $w$ and $c$ are interchanged. For the sake of brevity, if no confusion arises, we identify $\mathfrak{B}$ and $\mathfrak{B}_{(w, c)}^{*}$ and denote $\mathfrak{B}_{w}:=\pi_{w}\left(\mathfrak{B}_{(w, c)}\right)$ and $\mathfrak{B}_{c}:=\pi_{c}\left(\mathfrak{B}_{(w, c)}\right)$.

Definition 4. Given a behavior $\mathfrak{B}_{(w, c)} \subset \mathcal{U}^{\mathrm{w}+\mathrm{c}}$ we say that $c$ is observable from $w$ if $\left(w, c_{1}\right),\left(w, c_{2}\right) \in \mathfrak{B}_{(w, c)}$ implies $c_{1}=c_{2}$.

Usually, in control problems involving behaviors with two types of variables it is important to consider the set of variables that are not observable or hidden from the remaining set of variables, see $[15,16,17]$. Hence, given a behavior $\mathfrak{B}_{(w, c)}$ we shall define

$$
\mathfrak{B}_{(0, c)}:=\left\{c \in \mathcal{U}^{\mathrm{c}} \mid(0, c) \in \mathfrak{B}_{(w, c)}\right\},
$$

as the behavior of the variables $c$ that are not observable or hidden from $w$. Clearly, if $\mathfrak{B}_{(w, c)}=\operatorname{ker}[R M]$ then $\mathfrak{B}_{(0, c)}=\operatorname{ker} M$. Similarly we define $\mathfrak{B}_{(w, 0)}$ as the set of $w$ variables that are hidden from the variables $c$. Taking into account that we are dealing with linear behaviors, it is not difficult to verify that $c$ is observable from $w$ 
if and only if $\mathfrak{B}_{(0, c)}$ is the zero behavior. Similarly, $w$ is observable from $c$ if and only if $\mathfrak{B}_{(w, 0)}$ is the zero behavior.

\section{Implementation}

The behavioral approach to control rests on the basic idea that to control a system is to impose appropriate additional restrictions to its variables in order to obtain a new desired behavior. These additional restrictions are achieved by interconnecting the given system with another system called the controller. From the mathematical point of view, system interconnection corresponds to the intersection of the behavior to be controlled with the controller behavior.

Two situations have been considered in the literature. The first one is known as full interconnection and corresponds to the case where the controller is allowed to impose restrictions on all the system variables. The second,called partial interconnection, considers interconnections where one is only allowed to use some of the system variables for the purpose of interconnection.

\subsection{Control by regular full interconnection}

The full interconnection of a behavior to be controlled, $\mathfrak{B} \subset \mathcal{U}^{\mathrm{w}}$, with a controller behavior, $\mathcal{C} \subset \mathcal{U}^{\mathrm{w}}$, yields a controlled behavior given by

$$
\mathcal{K}=\mathfrak{B} \cap \mathcal{C},
$$

or alternatively, in module terms, by $\operatorname{Mod}(\mathcal{K})=\operatorname{Mod}(\mathfrak{B})+\operatorname{Mod}(\mathcal{C})$. If (3) holds, we say that $\mathcal{K}$ is implementable by full interconnection from $\mathfrak{B}$.

A particular interesting type of interconnection corresponds to the case where the restrictions imposed by the controller do not overlap with the restrictions already active for the behavior to be controlled. Recalling that the elements of the modules associated with a behavior represent the corresponding equations (or restrictions), this means, in terms of the corresponding modules that

$$
\operatorname{Mod}(\mathfrak{B}) \cap \operatorname{Mod}(\mathcal{C})=\{0\},
$$

(or, equivalently, that $\mathfrak{B}+\mathcal{C}=\mathcal{U}^{\mathrm{w}}$ ) and therefore

$$
\operatorname{Mod}(\mathcal{K})=\operatorname{Mod}(\mathfrak{B}) \oplus \operatorname{Mod}(\mathcal{C})
$$

In this case we say that the interconnection of $\mathfrak{B}$ and $\mathcal{C}$ is a regular interconnection and denote it by $\mathfrak{B} \cap$ reg $\mathrm{C}$. For a $1 \mathrm{D}$ behaviors, we know from the work of Willems [18] that controllability is equivalent to implementation of any sub-behavior by 
means of regular interconnection. Again the situation for $\mathrm{nD}$ behaviors is more involved. The following necessary (and not necessarily sufficient) condition for implementation of $\mathrm{nD}$ behaviors by regular interconnection has been derived in [14, Th. 4.5, p. 124].

Theorem 2. Let $\mathfrak{B}$ and $\mathcal{K}$ be two $n D$ behaviors and $\mathfrak{B}^{c}$ the controllable part of $\mathfrak{B}$. Then if $\mathcal{K}$ is implementable by regular interconnection from $\mathfrak{B}$ then $\mathfrak{B}=\mathfrak{B}^{c}+\mathcal{K}$.

This result can be intuitively explained by the fact that an autonomous part of a behavior may be somehow considered as obstructions to the (regular) control of that behavior, as happens for instance with the non-controllable modes in the context of pole-placement for classical state-space systems. Using this result it is possible to show the next useful Lemma.

Lemma 1. [4, Lemma 6] Let $\mathfrak{B}$ and $\mathcal{C}$ be two $n D$ behaviors. If the interconnection of $\mathfrak{B}$ and $\mathcal{C}$ is regular then so is the interconnection between $\mathfrak{B}^{c}$ and $\mathcal{C}$.

Proof. Let $\mathfrak{B} \cap \mathcal{C}=\mathcal{K}$ with regular interconnection, i.e., $\operatorname{Mod}(\mathfrak{B}) \oplus \operatorname{Mod}(\mathcal{C})=$ $\operatorname{Mod}(\mathcal{K})$. Using Theorem 2 we have that $\mathfrak{B}=\mathfrak{B}^{c}+\mathcal{K}$ or equivalently $\operatorname{Mod}(\mathfrak{B})=$ $\operatorname{Mod}\left(\mathfrak{B}^{c}\right) \cap \operatorname{Mod}(\mathcal{K})=\operatorname{Mod}\left(\mathfrak{B}^{c}\right) \cap(\operatorname{Mod}(\mathfrak{B}) \oplus \operatorname{Mod}(\mathcal{C}))$. Using that $\operatorname{Mod}(\mathfrak{B}) \subset$ $\operatorname{Mod}\left(\mathfrak{B}^{c}\right)$ one easily show that $\operatorname{Mod}\left(\mathfrak{B}^{c}\right) \cap(\operatorname{Mod}(\mathfrak{B}) \oplus \operatorname{Mod}(\mathcal{C}))=\left(\operatorname{Mod}\left(\mathfrak{B}^{c}\right) \cap\right.$ $\operatorname{Mod}(\mathcal{C})) \oplus \operatorname{Mod}(\mathfrak{B})$. Since $\operatorname{Mod}(\mathfrak{B}) \cap \operatorname{Mod}(\mathcal{C})=\{0\}$ we have that $\operatorname{Mod}\left(\mathfrak{B}^{c}\right) \cap$ $\operatorname{Mod}(\mathcal{C})=\{0\}$.

Lemma 1 shows that the controllable part of a behavior plays an important role in the context of regular interconnections. Indeed, a controller which does not interconnect with $\mathfrak{B}^{c}$ in a regular way, can not interconnect with $\mathfrak{B}$ regularly.

Next we present a more surprising result, proven in [5, Theorem 18], that shows that the possibility of implementing autonomous sub-behaviors of $\mathfrak{B}$ by regular interconnection may also impose conditions in the controllable part of $\mathfrak{B}$, depending on the autonomy degree of such sub-behaviors. We shall include its short proof for the sake of completeness.

Theorem 3. Let $\mathfrak{B}$ be a behavior. If $\mathcal{K} \subset \mathfrak{B}$ is regularly implementable from $\mathfrak{B}$ and has autonomy degree larger than 1 then $\mathfrak{B}^{c}$ (the controllable part of $\mathfrak{B}$ ) is rectifiable.

Proof. In order to prove the result we will make use of the duality between $\mathfrak{B}$ and $\operatorname{Mod}(\mathfrak{B})$. Obviously, $\mathfrak{B} \cap_{\text {reg }} \mathcal{C}=\mathcal{K}$ if and only if $\operatorname{Mod}(\mathfrak{B}) \oplus \operatorname{Mod}(\mathcal{C})=\operatorname{Mod}(\mathcal{K})$. The assumption that $\mathcal{K}$ has autonomy degree $\geq 2$ amounts to saying that the height of the annihilator of $\mathbb{C}^{q}\left[\underline{s}, \underline{s}^{-1}\right] /(\operatorname{Mod}(\mathfrak{B}) \oplus \operatorname{Mod}(\mathcal{C}))$ is $\geq 2$, see [22, Lemma 4.7, p. 54]. Equivalently, the annihilator of $\mathbb{C}^{q}\left[\underline{s}, \underline{s}^{-1}\right] /(\operatorname{Mod}(\mathfrak{B}) \oplus \operatorname{Mod}(\mathcal{C}))$ contains at least two coprime elements, see [22, Lemma 3.6].

Further, the interconnection $\mathfrak{B} \cap \mathcal{C}$ is regular if and only if $\mathfrak{B}^{c} \cap \mathcal{C}^{c}$ is regular, where $\mathfrak{B}^{c}$ and $\mathcal{C}^{c}$ denote the corresponding controllable parts, see [6, Lemma 12]. Obviously $\mathfrak{B}^{c} \cap \mathfrak{C}^{c} \subset \mathfrak{B} \cap \mathcal{C}$ and therefore autodeg $\left(\mathfrak{B}^{c} \cap \mathfrak{C}^{c}\right) \geq \operatorname{autodeg}(\mathfrak{B} \cap \mathcal{C})$. 
Thus we have, by assumption, that the annihilator of $\mathbb{C}^{q}\left[\underline{s}, \underline{s}^{-1}\right] /\left(\operatorname{Mod}\left(\mathfrak{B}^{c}\right) \oplus\right.$ $\left.\operatorname{Mod}\left(\mathrm{C}^{c}\right)\right)$ contains at least two coprime elements, say $d_{1}, d_{2}$. Note that since $\mathfrak{B}^{c}$ and $\mathcal{C}^{c}$ are controllable, $\mathbb{C}^{q}\left[\underline{s}, \underline{s}^{-1}\right] / \operatorname{Mod}\left(\mathfrak{B}^{c}\right)$ and $\mathbb{C}^{q}\left[\underline{s}, \underline{s}^{-1}\right] / \operatorname{Mod}\left(\mathcal{C}^{c}\right)$ are torsion free.

We prove that $\mathfrak{B}^{c}$ is rectifiable by showing that $\mathbb{C}^{q}\left[\underline{s}, \underline{s}^{-1}\right] / \operatorname{Mod}\left(\mathfrak{B}^{c}\right)$ is free as a $\mathbb{R}\left[\underline{s}, \underline{s}^{-1}\right]$-module.

Consider an element $\xi \in \mathbb{C}^{q}\left[\underline{s}, \underline{s}^{-1}\right]$. There are coprime elements $d_{1}, d_{2}$ with $d_{1} \xi=a_{1}+b_{1}, d_{2} \xi=a_{2}+b_{2}$ with $a_{1}, a_{2} \in \operatorname{Mod}\left(\mathfrak{B}^{c}\right), b_{1}, b_{2} \in \operatorname{Mod}\left(\mathfrak{C}^{c}\right)$. The element $\tau_{1}=\frac{a_{1}}{d_{1}}=\frac{a_{2}}{d_{2}} \in \mathbb{C}^{q}\left(\underline{s}, \underline{s}^{-1}\right)$ has the property $d_{1} \tau_{1}, d_{2} \tau_{1} \in \mathbb{C}^{q}\left[\underline{s}, \underline{s}^{-1}\right]$, where $\mathbb{C}^{q}\left(\underline{s}, \underline{s}^{-1}\right)$ stands for the field of rational Laurent polynomials. Since $d_{1}, d_{2}$ are coprime, this implies that $\tau_{1} \in \mathbb{C}^{q}\left[\underline{s}, \underline{s}^{-1}\right]$. Since $\mathbb{C}^{q}\left[\underline{s}, \underline{s}^{-1}\right] / \operatorname{Mod}\left(\mathfrak{B}^{c}\right)$ has no torsion, one obtains $\tau_{1} \in \operatorname{Mod}\left(\mathfrak{B}^{c}\right)$.

The same argument shows that $\tau_{2}=\frac{b_{1}}{d_{1}}=\frac{b_{2}}{d_{2}}$ belongs to $\operatorname{Mod}\left(\mathrm{C}^{c}\right)$. Hence $\xi=\tau_{1}+$ $\tau_{2} \in \operatorname{Mod}\left(\mathfrak{B}^{c}\right) \oplus \operatorname{Mod}\left(\complement^{c}\right)$ and $\mathbb{C}^{q}\left[\underline{s}, \underline{s}^{-1}\right]=\operatorname{Mod}\left(\mathfrak{B}^{c}\right) \oplus \operatorname{Mod}\left(\complement^{c}\right)$. Then $\operatorname{Mod}\left(\mathfrak{B}^{c}\right)$ and $\operatorname{Mod}\left(\mathbb{C}^{c}\right)$ are projective modules and therefore free. Finally, since $\operatorname{Mod}\left(\mathbb{C}^{c}\right) \approx$ $\mathbb{C}^{q}\left[\underline{s}, \underline{s}^{-1}\right] / \operatorname{Mod}\left(\mathfrak{B}^{c}\right)$ one obtains that $\mathbb{C}^{q}\left[\underline{s}, \underline{s}^{-1}\right] / \operatorname{Mod}\left(\mathfrak{B}^{c}\right)$ is free. This concludes the proof.

One can conclude from Theorem 3 that, in contrast to the 1D case, regular implementability is a very restrictive property in the context of $\mathrm{nD}$ behaviors (with $n \geq 2$ ).

When the controllable part of $\mathfrak{B}$ is rectifiable, it is possible to further exploit the simplified form of the rectified behavior in order to derive the following result on the autonomous-controllable decomposition of $\mathfrak{B}$.

Theorem 4. [5, Prop. 4] Let $\mathfrak{B}$ be a behavior with rectifiable controllable part. Then, there always exists an autonomous sub-behavior $\mathfrak{B}^{a}$ of $\mathfrak{B}$ such that $\mathfrak{B}=$ $\mathfrak{B}^{c} \oplus \mathfrak{B}^{a}$.

\subsection{Control by regular partial interconnection}

In the case of partial interconnection, one starts from a full behavior $\mathfrak{B}_{(w, c)}$, where $w$ is the variable to be controlled and $c$ is the control variable. The goal is to find a control variable behavior $\mathcal{C}$ whose interconnection with $\mathfrak{B}_{(w, c)}$ yields a desired behavior, $\mathcal{K}$ for the variable $w$. This can be formulated as finding $\mathcal{C}$ such that:

$$
\mathcal{K}=\pi_{w}\left(\mathfrak{B}_{(w, c)} \cap \mathcal{C}_{(w, c)}^{*}\right) .
$$

For simplicity of notation we shall write $\mathcal{C}_{c}$ or instead of $\mathcal{C}_{(w, c)}^{*}$; moreover we shall skip the subscript with the indication of the variable (and write, for instance, $\mathcal{C}$ and $\mathfrak{B}$ instead of $\mathcal{C}_{c}$ and $\mathfrak{B}_{w}$, respectively) if no confusion arises. 
Also in this context regularity plays an important role. Given two behaviors $\mathfrak{B}_{(w, c)} \subset \mathcal{U}^{\mathrm{w}+\mathrm{c}}$ and $\mathcal{C} \subset \mathcal{U}^{\mathrm{c}}$, we say that the interconnection $\mathfrak{B}_{(w, c)} \cap \mathcal{C}_{(w, c)}^{*}$ is regular if

$$
\operatorname{Mod}\left(\mathfrak{B}_{(w, c)}\right) \cap \operatorname{Mod}\left(\mathcal{C}_{(w, c)}^{*}\right)=\{0\},
$$

or equivalently if $\mathfrak{B}_{(w, c)}+\mathfrak{C}_{(w, c)}^{*}=\mathcal{U}^{\mathrm{w}+c}$. In this case, we denote the interconnection by $\mathfrak{B}_{(w, c)} \cap_{\text {reg }} \mathcal{C}_{(w, c)}^{*}$ or (in simplified notation) by $\mathfrak{B}_{(w, c)} \cap_{\text {reg }} \mathcal{C}$. Obviously, if $\mathcal{C}=\operatorname{ker} C, \operatorname{Mod}\left(\mathcal{C}_{(w, c)}^{*}\right)=\operatorname{RM}\left(\left[\begin{array}{ll}0 & C\end{array}\right]\right)$ and when no confusion arises we write $\operatorname{Mod}\left(\mathcal{C}_{(w, c)}^{*}\right)=\operatorname{Mod}(\mathcal{C})$.

The following lemma presents some interesting results about partial interconnections and hidden behaviors that can be found in [7, Lemma 9] or in [15, Corollary 14].

Lemma 2. Let $\mathfrak{B}_{(w, c)} \subset \mathcal{U}^{\mathrm{w}+\mathrm{c}}$ and $\mathcal{C} \subset \mathcal{U}^{\mathrm{c}}$ be two behaviors. Then, the following hold true.

1. $\pi_{w}\left(\mathfrak{B}_{(w, c)} \cap \mathcal{C}\right)=\pi_{w}\left(\mathfrak{B}_{(w, c)} \cap\left(\mathcal{C}+\mathfrak{B}_{(0, c)}\right)\right)$

2. $\mathfrak{B}_{(w, c)} \cap_{\text {reg }} \mathcal{C}$ if and only if $\mathfrak{B}_{(w, c)} \cap_{\text {reg }}\left(\mathcal{C}+\mathfrak{B}_{(0, c)}\right)$.

3. $\mathfrak{B}_{(w, c)} \cap_{\text {reg }} \mathrm{C}$ if and only if $\mathfrak{B}_{c} \cap_{\text {reg }} \mathrm{C}$.

Proof. Let $\mathfrak{B}_{(w, c)}=\operatorname{ker}[R \quad M]$ and $\mathcal{C}=\operatorname{ker} C$. Note that $\mathfrak{B}_{(0, c)}=\operatorname{ker} M \subset \mathcal{U}^{c}$ and since $\mathfrak{B}_{(0, c)} \subset \mathcal{C}+\mathfrak{B}_{(0, c)}$, then $\mathrm{C}+\mathfrak{B}_{(0, c)}=$ ker $K M$ for some L-polynomial matrix $K$.

1. It is enough to show that $\pi_{w}\left(\mathfrak{B}_{(w, c)} \cap\left(\mathcal{C}+\mathfrak{B}_{(0, c)}\right)\right) \subset \pi_{w}\left(\mathfrak{B}_{(w, c)} \cap \mathcal{C}\right)$ since the other inclusion is trivial. Let $w \in \pi_{w}\left(\mathfrak{B}_{(w, c)} \cap\left(\mathcal{C}+\mathfrak{B}_{(0, c)}\right)\right)$. Then, by definition of $\pi_{w}$ there exists a $c$ such that $(w, c) \in \mathfrak{B}_{(w, c)} \cap\left(\mathcal{C}+\mathfrak{B}_{(0, c)}\right)=\operatorname{ker}\left[\begin{array}{cc}R & M \\ 0 & K M\end{array}\right]$. Clearly, $c$ must satisfy $K M c=0$, i.e., $c \in \mathcal{C}+\mathfrak{B}_{(0, c)}=\operatorname{ker} K M$ and therefore $c=c^{*}+c^{* *}$, where $c^{*} \in \mathcal{C}$ and $c^{* *} \in \mathfrak{B}_{(0, c)}=\operatorname{ker} M$. Hence, as $(w, c) \in \operatorname{ker}\left[\begin{array}{ll}R & M\end{array}\right],\left(w, c^{*}\right) \in$ $\operatorname{ker}\left[\begin{array}{ll}R & M\end{array}\right]$ which implies that $\left(w, c^{*}\right) \in \operatorname{ker}\left[\begin{array}{cc}R & M \\ 0 & C\end{array}\right]=\mathfrak{B}_{(w, c)} \cap \mathcal{C}$, and therefore $w \in$ $\pi_{w}\left(\mathfrak{B}_{(w, c)} \cap \mathcal{C}\right)$.

2.By Theorem 1, the proof of 3 amounts to showing that

$\operatorname{RM}\left(\left[\begin{array}{ll}R & M\end{array}\right]\right) \cap \operatorname{RM}\left(\left[\begin{array}{ll}0 & C\end{array}\right]\right)=\{0\}$ if and only if $\operatorname{RM}\left(\left[\begin{array}{ll}R & M\end{array}\right]\right) \cap \operatorname{RM}\left(\left[\begin{array}{ll}0 & K M\end{array}\right]\right)=\{0\}$.

As ker $C=\mathcal{C} \subset \mathcal{C}+\mathfrak{B}_{(0, c)}=\operatorname{ker} K M, \operatorname{RM}(K M) \subset \operatorname{RM}(C)$ and the "only if" part is obvious. For the converse, let $(0,0) \neq(r, m) \in \operatorname{RM}\left(\left[\begin{array}{ll}R & M\end{array}\right]\right) \cap \operatorname{RM}\left(\left[\begin{array}{ll}0 & C\end{array}\right]\right)$. Clearly $r$ must be zero and then there exists an L-polynomial row $s$ such that $s\left[\begin{array}{ll}R & M\end{array}\right]=(0, m) \neq(0,0)$, which implies $s M=m \in \mathrm{RM}(C) \cap \mathrm{RM}(M)=\mathrm{RM}(K M)$. Thus, $(0, m) \in \operatorname{RM}\left(\left[\begin{array}{ll}R & M\end{array}\right]\right) \cap \operatorname{RM}\left(\left[\begin{array}{ll}0 & K M\end{array}\right]\right)$.

3. In terms of the corresponding modules we need to show that

$$
\operatorname{RM}\left(\left[\begin{array}{ll}
R & M
\end{array}\right]\right) \cap \operatorname{RM}\left(\left[\begin{array}{ll}
0 & C
\end{array}\right]\right)=\{0\} \text { if and only if } \operatorname{RM}(L M) \cap \operatorname{RM}(C)=\{0\},
$$

where $L$ is an MLA of $R$. In order to prove the "if" part, let $(0,0) \neq(r, m) \in$ $\operatorname{RM}\left(\left[\begin{array}{ll}R & M\end{array}\right]\right) \cap \operatorname{RM}\left(\left[\begin{array}{ll}0 & C\end{array}\right]\right)$. It is easy to see that $r$ must be zero and therefore there 
exists $s \in L$ such that $s\left[\begin{array}{ll}R & M\end{array}\right]=(0, m)$. Thus, $0 \neq s M=m \in \operatorname{RM}(L M) \cap \operatorname{RM}(C)$. To prove the converse implication suppose that $0 \neq m \in \mathrm{RM}(L M) \cap \mathrm{RM}(C)$. Then, $m=\alpha L M=\beta C$ for some L-polynomial rows $\alpha$ and $\beta$. This implies that $(0, m)=$ $\alpha L\left[\begin{array}{ll}R & M\end{array}\right]=\beta\left[\begin{array}{ll}0 & C\end{array}\right]$ and therefore $(0,0) \neq(0, m) \in \operatorname{RM}\left(\left[\begin{array}{ll}R & M\end{array}\right]\right) \cap \operatorname{RM}\left(\left[\begin{array}{ll}0 & C\end{array}\right]\right)$.

A behavior $\mathcal{K} \subset \mathcal{U}^{\mathrm{w}}$ is trivially implementable from a given behavior $\mathfrak{B} \subset \mathcal{U}^{\mathrm{w}}$ by full (not necessarily regular) interconnection if and only if $\mathcal{K} \subset \mathfrak{B}$. This condition is however not enough in the partial interconnection case. Indeed, it was proven in [1, $15,16]$ that $\mathcal{K}$ is implementable by partial (not necessarily regular) interconnection from $\mathfrak{B}_{(w, c)}$ if and only if

$$
\mathfrak{B}_{(w, 0)} \subset \mathcal{K} \subset \mathfrak{B}_{w}=\pi_{w}\left(\mathfrak{B}_{(w, c)}\right) .
$$

For regular partial interconnections the implementation problem was fully addressed and solved in the 1D context in [1]. In effect, the following necessary and sufficient conditions for the regular implementation of a behavior $\mathcal{K}$ were given:

1. $\mathcal{K}$ is implementable by partial interconnection, i.e., $\mathfrak{B}_{(w, 0)} \subset \mathcal{K} \subset \mathfrak{B}_{w}$,

2. $\mathcal{K}+\mathfrak{B}_{w}^{c}=\mathfrak{B}_{w}$, where $\mathfrak{B}_{w}^{c}$ stands for the controllable part of $\mathfrak{B}_{w}$.

Note that the second condition is equivalent (in the $1 \mathrm{D}$ case) to $\mathcal{K}$ being regularly implementable by full interconnection from $\mathfrak{B}_{w}$. It was shown in $[15,13]$ that these two conditions were neither necessary nor sufficient in the $\mathrm{nD}$ case. Next we investigate when similar conditions hold in terms of the associated hidden behaviors. We say that a behavior is regular if admits a full row rank representation.

Theorem 5. Let $\mathcal{K} \subset \mathcal{U}^{\mathrm{w}}$ and $\mathfrak{B}_{(w, c)} \subset \mathcal{U}^{\mathrm{w}+c}$ be given. Assume that $\mathcal{K}$ is implementable by partial interconnection and that the hidden behavior $\mathfrak{B}_{(w, 0)}$ is regular. If $\mathcal{K}$ is regularly implementable by full interconnection (from $\mathfrak{B}_{w}$ ) then it is regularly implementable by partial interconnection.

Proof. Let $\left[\begin{array}{ll}\bar{R} & M\end{array}\right]$ be such that $\mathfrak{B}_{(w, c)}=\operatorname{ker}\left[\begin{array}{ll}\bar{R} & M\end{array}\right]$. Since $\mathfrak{B}_{(w, 0)}=\operatorname{ker} \bar{R}$ is regular we can assume without loss of generality that $\bar{R}=\left[\begin{array}{c}R \\ 0\end{array}\right]$ with $R$ full row rank and therefore $\mathfrak{B}_{(w, c)}=\operatorname{ker}\left[\begin{array}{ll}R & M_{1} \\ 0 & M_{2}\end{array}\right]$, for a suitable partition of $M$. Then, $\mathfrak{B}_{w}=$ $\pi_{w}\left(\mathfrak{B}_{(w, c)}\right)=\operatorname{ker} X R$, where $\left[\begin{array}{ll}X & Y\end{array}\right]$ is an MLA of $\left[\begin{array}{l}M_{1} \\ M_{2}\end{array}\right]$. Let $\overline{\mathcal{C}}=\operatorname{ker} C \subset \mathcal{U}^{w}$ be the controller that implements $\mathcal{K}$ by full interconnection. As $\mathcal{K}$ is implementable by partial interconnection, $\mathfrak{B}_{(w, 0)} \subset \mathcal{K} \subset \overline{\mathcal{C}}$ it follows that there exists a matrix $L$ such that $C=L R$. Take $\mathcal{C}=\operatorname{ker} L M_{1} \subset \mathcal{U}^{\mathrm{c}}$. Next we show that $\mathcal{C}$ regularly implements $\mathcal{K}$ by partial interconnection. It is easy to check that $\mathcal{C}$ implements $\mathcal{K}$. To show that the interconnection is regular suppose that the row vector $m$ belongs to $R M\left(\left[\begin{array}{ll}0 & L M_{1}\end{array}\right]\right) \cap R M\left(\left[\begin{array}{ll}R & M_{1} \\ 0 & M_{2}\end{array}\right]\right)$. This means that there exist row vectors $s$, and $t=\left[\begin{array}{ll}t_{1} & t_{2}\end{array}\right]$ such that $m=s\left[\begin{array}{ll}0 & L M_{1}\end{array}\right]=\left[\begin{array}{ll}t_{1} & t_{2}\end{array}\right]\left[\begin{array}{cc}R & M_{1} \\ 0 & M_{2}\end{array}\right]$. As $R$ is full row rank $t_{1}=0$. 
This implies that $\left[s L \quad-t_{2}\right]\left[\begin{array}{l}M_{1} \\ M_{2}\end{array}\right]=0$, and hence $\left[\begin{array}{ll}s L & -t_{2}\end{array}\right]=v\left[\begin{array}{ll}X & Y\end{array}\right]$ for some row vector $v$. In turn, this implies that $s L R=v X R$. As, by assumption, the interconnection of $\mathfrak{B}_{w}=\operatorname{ker} X R$ and $\overline{\mathrm{C}}=\operatorname{ker} L R$ is regular, $s L R=(v X R=) 0$, and, since $R$ has full row rank, $s L=0$. Therefore $m=s\left[\begin{array}{ll}0 & L M_{1}\end{array}\right]=0$, which concludes the proof.

Using part 3 of Lemma 2, and applying the same type of reasoning as in the proof of Theorem 5, one can derive the following corollary.

Corollary 1. Let $\mathcal{K} \subset \mathcal{U}^{\mathrm{w}}$ and $\mathfrak{B}_{(w, c)} \subset \mathcal{U}^{\mathrm{w}+c}$ be given. Assume that $\mathcal{K}$ is implementable by partial interconnection and that the hidden behavior $\mathfrak{B}_{(0, c)}$ is regular. If $\mathcal{K}$ is regularly implementable by partial interconnection then it is regularly implementable by full interconnection (from $\mathfrak{B}_{w}$ ).

Remark 1. Note that rectifiable behaviors admit a full row rank representation, i.e., are regular, and therefore Theorem 5 and Corollary 1 are still valid if we assume that $\mathfrak{B}_{(w, 0)}$ and $\mathfrak{B}_{(0, c)}$ respectively are rectifiable. Moreover, in the $2 \mathrm{D}$ case one can assume controllability instead of rectifiability as controllable behaviors always have a full row rank representation.

\subsection{Controllers}

In this section we look at a special behavior that has also been introduced in $[2,16,19]$ under the name of canonical controller. In particular, we study its effectiveness in solving partial control problems - a question which has also been considered in $[3,19]$ for the 1D case - and generalize the corresponding 1D results to the $\mathrm{nD}$ case. We conclude the section by analyzing the performance of regular controllers in this context. The results of this section (except for Theorem 10) were first presented in [13] although some can also be found in [15] in a more moduletheoretical framework.

It is immediately apparent that the study of partial control problems requires additional tools with respect to full control problems. For this reason, it is desirable to translate partial control problems into full control ones. In the 1D case, it is possible to make this translation in terms of full control problems for behaviors involving only the to-be-controlled variable $w$. Unfortunately this is no longer true in the higher dimensional $(\mathrm{nD})$ case. Therefore we shall try to characterize regular implementation (by partial control) in terms of conditions on the control variable behavior, rather than by means of conditions on the behavior of the variables to be controlled. To this end we introduce the notion of canonical controller associated to a given control problem. For a given control objective $\mathcal{K} \subset \mathcal{U}^{\mathrm{w}}$, the canonical controller associate with $\mathcal{K}$ is defined as follows:

$$
\mathcal{C}^{\text {can }}(\mathcal{K}):=\left\{c \mid \exists w \text { such that }(w, c) \in \mathfrak{B}_{(w, c)} \text { and } w \in \mathcal{K}\right\}
$$


For simplicity we use $e^{\text {can }}$ for $\mathcal{C}^{\text {can }}(\mathcal{K})$. Thus, the canonical controller consists of all the control variable trajectories compatible with the desired behavior for the variables to be controlled.

We start by relating the implementation of $\mathcal{K}$ from $\mathfrak{B}_{(w, c)}$ (by partial control) with the implementation of the corresponding canonical controller from $\mathfrak{B}_{c}$. First we treat the implementation problem and then the regular implementation.

Theorem 6. Given a plant behavior $\mathfrak{B}_{(w, c)}$ and an implementable control objective $\mathcal{K}$, the following holds.

1. If the controller $\mathcal{C}$ implements $\mathcal{C}^{\text {can }}$ from $\mathfrak{B}_{c}$ by full control, then it implements $\mathcal{K}$ from $\mathfrak{B}_{(w, c)}$.

2. If the controller $\tilde{\mathcal{C}}$ implements $\mathcal{K}$ from $\mathfrak{B}_{(w, c)}$, then the controller $\tilde{\mathfrak{C}}+\mathfrak{B}_{(0, c)}$ implements $\mathfrak{C}^{\text {can }}$ from $\mathfrak{B}_{c}$ by full control.

Proof. Let $R w=M c$ be a representation of $\mathfrak{B}_{(w, c)}$. Then, $\mathfrak{B}_{c}=\operatorname{ker} N M$, where $N$ be an $\mathrm{nD}$ polynomial matrix which is an minimal left annihilator (MLA) of $R$. To prove the first statement assume that the controller $\mathcal{C}=\operatorname{ker} K$ implements $\mathcal{C}^{\text {can }}$ and apply this controller to the plant. This yields the $(w, c)$-behavior described by the equations:

$$
\left\{\begin{array}{l}
R w=M c \\
0=K c .
\end{array}\right.
$$

We next show that the corresponding $w$-behavior coincides with $\mathcal{K}$, which clearly implies that $\mathcal{C}$ implements $\mathcal{K}$ from $\mathfrak{B}_{(w, c)}$.

Suppose then that $w^{*}$ belongs to the $w$-behavior induced by equations (4), i.e., that there exists $c^{*}$ such that the pair $\left(w^{*}, c^{*}\right)$ satisfies these equations. This implies that $c^{*} \in \mathfrak{B}_{c} \cap \mathcal{C}=\mathcal{C}^{c a n}$ and hence, by the definition of the canonical controller, there exists $\bar{w} \in \mathcal{K}$ such that $(\bar{w}, c) \in \mathfrak{B}_{(w, c)}$. Thus, by linearity, $\left(w^{*}-\bar{w}, 0\right) \in \mathfrak{B}_{(w, c)}$, meaning that $w^{*}-\bar{w} \in \mathfrak{B}_{(w, 0)}$. Since $\mathcal{K}$ is by assumption implementable, $\mathfrak{B}_{(w, 0)} \subset \mathcal{K}$ and $w^{*}-\bar{w} \in \mathcal{K}$. Consequently also $w^{*} \in \mathcal{K}$ and therefore the $w$-behavior induced by equations (4) is contained in $\mathcal{K}$.

Conversely, suppose that $w^{*} \in \mathcal{K}$. Then obviously $w^{*} \in \mathfrak{B}_{w}$ and hence there exists $c^{*}$ such that $\left(w^{*}, c^{*}\right) \in \mathfrak{B}_{(w, c)}$, i.e., such that

$$
R w^{*}=M c^{*} .
$$

By the definition of the canonical controller, this means that $c^{*} \in \mathrm{C}^{\text {can }}$. Since $\mathrm{C}^{\text {can }}$ is assumed to be implementable by $\mathcal{C}, \mathcal{C}^{\text {can }} \subset \mathcal{C}$ and therefore $c^{*} \in \mathcal{C}$, i.e.,

$$
K c^{*}=0 .
$$

Thus, the pair $\left(w^{*}, c^{*}\right)$ satisfies equations (4), which means that $w^{*}$ is in the $w$ behavior induced by these equations. So, $\mathcal{K}$ is contained in that behavior. As mentioned before, this shows that $\mathcal{C}$ implements $\mathcal{K}$ from $\mathfrak{B}_{(w, c)}$.

As for the second statement assume now that the controller $\tilde{\mathcal{C}}=\operatorname{ker} K$ implements $\mathcal{K}$ from $\mathfrak{B}_{(w, c)}$. Let $c^{*} \in \mathcal{C}^{c a n}$. This means that there exists $w^{*}$ such that $\left(w^{*}, c^{*}\right) \in \mathfrak{B}_{(w, c)}$ 
and $w^{*} \in \mathcal{K}$. This last condition implies that there exists $\bar{c} \in \tilde{\mathcal{C}}$ such that $\left(w^{*}, \bar{c}\right) \in$ $\mathfrak{B}_{(w, c)}$. Note that by the linearity of $\mathfrak{B}_{(w, c)},\left(0, c^{*}-\bar{c}\right) \in \mathfrak{B}_{(w, c)}$; hence $c^{*}-\bar{c} \in \mathfrak{B}_{(0, c)}$ and therefore (taking into account that $\bar{c} \in \tilde{\mathcal{C}}$ ) we have that $c^{*} \in \mathfrak{B}_{(0, c)}+\tilde{\mathcal{C}}$. Thus, $\mathrm{e}^{\text {can }} \subset \mathfrak{B}_{(0, c)}+\tilde{\mathcal{C}}$ and, since also $\mathrm{e}^{\text {can }} \subset \mathfrak{B}_{c}$, e $^{\text {can }} \subset\left(\mathfrak{B}_{(0, c)}+\tilde{\mathcal{C}}\right) \cap \mathfrak{B}_{c}$.

Conversely, assume that $c^{*} \in\left(\mathfrak{B}_{(0, c)}+\tilde{\mathcal{C}}\right) \cap \mathfrak{B}_{c}$. Then, there exist $w^{*}$ and $\bar{c} \in \tilde{\mathcal{C}}$ such that $\left(w^{*}, c^{*}\right) \in \mathfrak{B}_{(w, c)}, \bar{c} \in \tilde{\mathcal{C}}$ and $c^{*}-\bar{c} \in \mathfrak{B}_{(0, c)}$. This implies that $\left(w^{*}, \bar{c}\right) \in$ $\mathfrak{B}_{(w, c)}$ and, since $\tilde{\mathcal{C}}$ implements $\mathcal{K}$ from $\mathfrak{B}_{(w, c)}, w^{*} \in \mathcal{K}$. Together with the fact that $\left(w^{*}, c^{*}\right) \in \mathfrak{B}_{(w, c)}$, taking the definition of $\mathcal{C}^{c a n}$ into account, this allows to conclude that $c^{*} \in \mathcal{C}^{c a n}$. Therefore $\left(\mathfrak{B}_{(0, c)}+\tilde{\mathcal{C}}\right) \cap \mathfrak{B}_{c} \subset \mathcal{C}^{c a n}$. This finally proves that $\mathcal{e}^{c a n}=$ $\left(\mathfrak{B}_{(0, c)}+\tilde{\mathcal{C}}\right) \cap \mathfrak{B}_{c}$, which amounts to say that $\mathfrak{B}_{(0, c)}+\tilde{\mathcal{C}}$ implements $\mathcal{C}^{c a n}$ from $\mathfrak{B}_{c}$ by full control.

Note that, as a consequence of this theorem, if the hidden behavior $\mathfrak{B}_{(0, c)}=\{0\}$, then $\mathcal{C}$ implements $\mathcal{K}$ from $\mathfrak{B}_{(w, c)}$ if and only if it implements $\mathcal{C}^{\text {can }}$ from $\mathfrak{B}_{c}$ by full control.

Next we extend Theorem 6 for regular interconnections.

Theorem 7. Given a plant behavior $\mathfrak{B}_{(w, c)}$ and an implementable control objective $\mathcal{K}$, the following holds.

1. If the controller $\mathcal{C}$ implements $\mathfrak{e}^{\text {can }}$ from $\mathfrak{B}_{c}$ by regular full control, then $\mathcal{C}$ implements $\mathcal{K}$ regularly from $\mathfrak{B}_{(w, c)}$.

2. If the controller $\tilde{\mathcal{C}}$ implements $\mathcal{K}$ regularly from $\mathfrak{B}_{(w, c)}$, then the controller $\tilde{\mathcal{C}}+$ $\mathfrak{B}_{(0, c)}$ implements $\mathrm{C}^{\text {can }}$ from $\mathfrak{B}_{c}$ by regular full control.

Proof. Since the statements about implementation have already been proven in Theorem 6 it now suffices to prove the statements concerning regularity.

To show the first statement let $r=\left[\begin{array}{ll}0 & \bar{r}\end{array}\right] \in \operatorname{Mod}\left(\mathfrak{B}_{(w, c)}\right) \cap \operatorname{Mod}\left(\mathcal{C}_{(w, c)}^{*}\right)$ (note that since $w$ is free in $\mathcal{C}_{(w, c)}^{*}$, the first components of $r$ must be zero). Then, clearly, $\bar{r} \in \operatorname{Mod}(\mathcal{C})$. Moreover, $\mathfrak{B}_{c} \subset \operatorname{ker} \bar{r}$, and hence $\bar{r} \in \operatorname{Mod}\left(\mathfrak{B}_{c}\right)$. Therefore $\bar{r} \in \operatorname{Mod}\left(\mathfrak{B}_{c}\right) \cap \operatorname{Mod}(\mathcal{C})$. In this way, if $\operatorname{Mod}\left(\mathfrak{B}_{(w, c)}\right) \cap \operatorname{Mod}\left(\mathcal{C}_{(w, c)}^{*}\right)$ has a nonzero element $r=\left[\begin{array}{ll}0 & \bar{r}\end{array}\right]$ with $\bar{r} \neq 0$ then also $\operatorname{Mod}\left(\mathfrak{B}_{c}\right) \cap \operatorname{Mod}(\mathcal{C})$ has a nonzero element $\bar{r}$, proving the desired implication. Statement 2. can be proved using similar arguments.

Again we remark that Theorem 7 implies that, in case $\mathfrak{B}_{(0, c)}=\{0\}$, $\mathcal{C}$ regularly implements $\mathcal{K}$ from $\mathfrak{B}_{(w, c)}$ if and only if it implements $\mathcal{e}^{c a n}$ from $\mathfrak{B}_{c}$ by regular full control.

Theorem 7 yields necessary and sufficient conditions for the problem of regular implementation by partial interconnections.

Corollary 2. Let $\mathfrak{B}_{(w, c)}$ be a given plant behavior and $\mathcal{K}$ a control objective. Assume further that $\mathcal{K}$ is implementable from $\mathfrak{B}_{(w, c)}$. Then $\mathcal{K}$ is regularly implementable from $\mathfrak{B}_{(w, c)}$ if and only if $\mathcal{C}^{\text {can }}$ is regularly implementable from $\mathfrak{B}_{c}$ by full control. 
In the previous considerations, the canonical controller associated to a given control problem has been considered as a control objective itself, whose ability to be implemented provides information on the possibility of implementing the true control objective. We now take a different perspective and consider the canonical controller in its most natural role, i.e., as being itself a controller. In this context, two questions obviously arise: Does the canonical controller implement the control objective? If so, is this implementation regular? The answers to these questions are given below.

Theorem 8. Given a plant behavior $\mathfrak{B}_{(w, c)}$, a control objective $\mathcal{K}$, let $\mathrm{C}^{\text {can }}$ be the associated canonical controller. Then, $\mathrm{e}^{\text {can }}$ implements $\mathcal{K}$ if and only of $\mathcal{K}$ is implementable.

Proof. The "only if" part of the statement is trivial. As for the "if" part, suppose that $\mathcal{K}$ is implementable, and let $\tilde{\mathcal{C}}=\operatorname{ker} K$ be a controller that implements this behavior. Then, by Theorem 6 , the controller $\tilde{\mathcal{C}}+\mathfrak{B}_{(0, c)}$ implements $\mathcal{\complement}^{\text {can }}$ from $\mathfrak{B}_{c}$. If $R w=M c$ is a representation of $\mathfrak{B}_{(w, c)}$ and $N$ is a MLA of $R, \mathfrak{B}_{(0, c)}=\operatorname{ker} M$ and $\mathfrak{B}_{c}=\operatorname{ker} N M$. Therefore, the fact that $\tilde{\mathcal{C}}+\mathfrak{B}_{(0, c)}$ implements ecan from $\mathfrak{B}_{c}$ means that $\mathrm{e}^{c a n}$ is the $c$-behavior induced by the following equations:

$$
\left\{\begin{array}{l}
N M c=0 \\
c=c_{1}+c_{2} \\
K c_{1}=0 \\
M c_{2}=0 .
\end{array}\right.
$$

Consequently, applying the canonical controller to the plant $\mathfrak{B}_{(w, c)}$ yields the restrictions:

$$
\left\{\begin{array}{l}
R w=M c \\
N M c=0 \\
c=c_{1}+c_{2} \\
K c_{1}=0 \\
M c_{2}=0,
\end{array}\right.
$$

that can easily be shown to have the same $w$-behavior as

$$
\left\{\begin{array}{l}
R w=M c_{1} \\
K c_{1}=0
\end{array}\right.
$$

But this $w$-behavior is precisely $\mathcal{K}$, which proves that $\mathcal{C}^{\text {can }}$ indeed implements $\mathcal{K}$.

Our last results concerns regular implementation by means of the canonical controller.

Theorem 9. Given a plant behavior $\mathfrak{B}_{(w, c)}$, a control objective $\mathcal{K}$, let $\mathcal{C}^{c a n}$ be the associated canonical controller. Then, $\mathcal{C}^{c a n}$ regularly implements $\mathcal{K}$ if and only of $\mathfrak{B}_{c}$ coincides with the whole c-trajectory universe, i.e., if and only if $\operatorname{Mod}\left(\mathfrak{B}_{\mathrm{c}}\right)=$ $\{0\}$.

Proof. Assume that $\mathfrak{e}^{\text {can }}$ regularly implements $\mathcal{K}$. Then, by Corollary $2, \mathfrak{e}^{\text {can }}+$ $\mathfrak{B}_{(0, c)}$ regularly implements $\mathcal{\complement}^{\text {can }}$ from $\mathfrak{B}_{c}$. This implies that $\operatorname{Mod}\left(\mathcal{C}^{\text {can }}+\mathfrak{B}_{(0, c)}\right) \cap$ 
$\operatorname{Mod}\left(\mathfrak{B}_{c}\right)=\{0\}$. But, as shown before, $\operatorname{Mod}\left(\mathrm{e}^{c a n}+\mathfrak{B}_{(0, c)}\right) \cap \operatorname{Mod}\left(\mathfrak{B}_{c}\right)=\operatorname{Mod}\left(\mathrm{e}^{c a n}\right) \cap$ $\operatorname{Mod}\left(\mathfrak{B}_{c}\right)$. As $\operatorname{Mod}\left(\mathfrak{B}_{c}\right) \subset \operatorname{Mod}\left(\mathcal{C}^{\text {can }}\right)$ (because $\left.\mathcal{\complement}^{c a n} \subset \mathfrak{B}_{c}\right)$, we obtain that $\operatorname{Mod}\left(\mathfrak{B}_{c}\right)=$ $\{0\}$.

Conversely, if $\operatorname{Mod}\left(\mathfrak{B}_{c}\right)=\{0\}$ then the canonical controller regularly implements itself from $\mathfrak{B}_{c}$. By Corollary 2 this implies that $\mathcal{C}^{c a n}$ also implements $\mathcal{K}$ regularly.

Corollary 3. The canonical controller is regular if and only if every controller is regular.

Proof. The if part is obvious. As for the only if part, we start by noting that, given a controller $\mathcal{C}, \operatorname{Mod}\left(\mathfrak{B}_{(w, c)}\right) \cap \operatorname{Mod}\left(\mathcal{C}_{(w, c)}^{*}\right)=\left\{r \mid r=\left[\begin{array}{ll}0 & \bar{r}\end{array}\right], \bar{r} \in \bmod (\mathcal{C}) \cap\right.$ $\left.\operatorname{Mod}\left(\mathfrak{B}_{c}\right)\right\}$. Assume now that the canonical controller is regular. Then, by the previous theorem, $\operatorname{Mod}\left(\mathfrak{B}_{c}\right)=\{0\}$ and consequently also $\operatorname{Mod}\left(\mathfrak{B}_{(w, c)}\right) \cap \operatorname{Mod}\left(\mathcal{C}_{(w, c)}^{*}\right)=$ $\{0\}$ for any given controller $\mathcal{C}$, which precisely means that the controller $\mathcal{C}$ is regular. This proves the desired result.

Theorems 8, 9 and Corollary 3 generalize the corresponding 1D results obtained in $[19,3]$ to the $\mathrm{nD}$ case.

Finally, we study another class of controllers that are of interest in the context of regular partial interconnections, namely, controllers that admit full row rank representations, called regular controllers. The regular implementation by means of a regular controller implies the regular implementation by full interconnection (from $\left.\mathfrak{B}_{w}\right)$.

Theorem 10. [4, Theorem 10] Let $\mathfrak{B}_{(w, c)}=\operatorname{ker}\left[\begin{array}{ll}R & M\end{array}\right]$ be a behavior. If a desired behavior $\mathcal{K}$ is implementable by regular partial interconnection with a regular controller $\mathcal{C}=\operatorname{ker}[0 \quad L M]$ then $\mathcal{K}=\mathfrak{B}_{w} \cap_{\text {reg }} \operatorname{ker}(L R)$, i.e., $\mathcal{K}$ can also be implementable by regular (full) interconnection from $\mathfrak{B}_{w}$.

Proof. Without loss of generality we supposed that the matrix $L M$ is full row rank since $\mathcal{C}$ is a regular behavior. Further, $\left[\begin{array}{cc}I & 0 \\ L & -I\end{array}\right] \cdot\left[\begin{array}{cc}R & M \\ 0 & L M\end{array}\right]=\left[\begin{array}{cc}R & M \\ L R & 0\end{array}\right]$. Let $X$ be the MLA of $M$. Hence $\Pi_{w}\left(\mathfrak{B}_{(w, c)} \cap \mathcal{C}\right)=\Pi_{w}\left(\operatorname{ker}\left[\begin{array}{cc}R & M \\ L R & 0\end{array}\right]\right)=\operatorname{ker}\left[\begin{array}{c}X R \\ L R\end{array}\right]$ $=\mathfrak{B}_{(w, c)} \cap \operatorname{ker} L R$. To see that the interconnection between $\mathfrak{B}_{(w, c)}$ and ker $L R$ is regular we prove that the interconnection between $\operatorname{ker}[R M] \cap \operatorname{ker}[L R \quad 0]$ is regular, i.e., $v\left[\begin{array}{ll}R & M\end{array}\right]=z\left[\begin{array}{ll}L R & 0\end{array}\right]$ for some row vectors $v$ and $z$, implies $v\left[\begin{array}{ll}R & M\end{array}\right]=0=z\left[\begin{array}{ll}L R & 0\end{array}\right]$. Suppose that $v\left[\begin{array}{ll}R & M\end{array}\right]=z\left[\begin{array}{ll}L R & 0\end{array}\right]$. Note that $z\left[\begin{array}{ll}L R & 0\end{array}\right]=z\left[\begin{array}{ll}0 & -L M\end{array}\right]+\left[\begin{array}{ll}L R & L M\end{array}\right]$ and then $v[R M]-z\left[\begin{array}{ll}L R & L M\end{array}\right]=(v-z L)[R M]=z\left[\begin{array}{ll}0 & -L M]\end{array}\right]$. By assumption that the intercon-

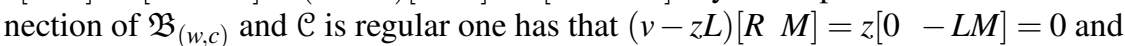
since $L M$ is full row rank one obtains that $z=0$ and therefore $v[R M]=z\left[\begin{array}{ll}L R & 0\end{array}\right]=0$ which proves that the interconnection is regular.

Acknowledgements The authors are supported by Portuguese funds through the CIDMA - Center for Research and Development in Mathematics and Applications, and the Portuguese Foundation for Science and Technology (FCT-Fundação para a Ciência e a Tecnologia), within project PEstUID/MAT/04106/2013. 


\section{References}

1. M.N. Belur and H.L. Trentelman. Stabilization, pole placement, and regular implementability. IEEE Trans. Automat. Control, 47(5):735-744, 2002.

2. A. Agung Julius, Jan Willem Polderman, and Arjan van der Schaft. Parametrization of the regular equivalences of the canonical controller. IEEE Trans. Automat. Control, 53(4), 2008.

3. A.A. Julius, J.C. Willems, M.N. Belur, and H.L. Trentelman. The canonical controllers and regular interconnection. Systems Control Lett., 54(8):787-797, 2005.

4. D. Napp and P. Rocha. Implementation of 2D strongly autonomous behaviors by full and partial interconnections. Lectures Notes in Control and Information Science (Springer), Vol. 389:369-378, 2009.

5. D. Napp and P. Rocha. Autonomous multidimensional systems and their implementation by behavioral control. Systems \& Control Letters, 59(3-4):203-208, 2010.

6. D. Napp and P. Rocha. Strongly autonomous interconnections and stabilization of 2D behaviors. Asian Journal of control, 12(2):127-135, 2010.

7. D. Napp and P. Rocha. Stabilization of discrete $2 \mathrm{~d}$ behaviors by regular partial interconnection. Mathematics of control signal and systems, Vol. 22 (4):295-316, 2011.

8. D. Napp, S. Shankar, and H.L. Trentelman. Regular implementation in the space of compactly supported functions. Systems and Control Letters, 57(10):851-855, 2008.

9. U. Oberst. Multidimensional constant linear systems. Acta Appl. Math., 20(1-2):1-175, 1990.

10. J.W. Polderman and I. Mareels. A behavioral approach to adaptive control. The Mathematics of Systems and Control: From Intelligent Control to Behavioral Systems, J.W. Polderman and H.L. Trentelman, eds., Foundation Systems and Control Groningen, The Netherlands, 1999.

11. J.W. Polderman and J.C. Willems. Introduction to Mathematical Systems Theory, A behavioral approach, volume 26 of Texts in Applied Mathematics. Springer-Verlag, New York, 1998.

12. C. Praagman, H.L. Trentelman, and R. Zavala Yoe. On the parametrization of all regularly implementing and stabilizing controllers. SIAM J. Control Optim., 45(6):2035-2053 (electronic), 2007.

13. P Rocha. Canonical controllers and regular implementation of $n \mathrm{D}$ behaviors. Proceedings of the 16th IFAC World Congress, 2005.

14. P. Rocha and J. Wood. Trajectory control and interconnection of $1 \mathrm{D}$ and $n \mathrm{D}$ systems. SIAM J. Control Optim., 40(1):107-134, 2001

15. H.L. Trentelman and D. Napp Avelli. On the regular implementability of $n \mathrm{D}$ systems. Systems and Control Letters, 56(4):265-271, 2007.

16. A.J. van der Schaft. Achievable behavior of general systems. Systems Control Lett., 49(2):141-149, 2003.

17. J.C. Willems. Paradigms and puzzles in the theory of dynamical systems. IEEE Trans. Automat. Control, 36(3):259-294, 1991.

18. J.C. Willems. On interconnections, control, and feedback. IEEE Trans. Automat. Control, 42(3):326-339, 1997

19. J.C. Willems, M.N. Belur, A.A. Julius, and H.L. Trentelman. The canonical controller and its regularity. Proceedings of the 42nd IEEE Conference on Decision and Control, Hawaii, USA, pages 1639-1644, 2003.

20. J.C. Willems and H.L. Trentelman. Synthesis of dissipative systems using quadratic differential forms. I. IEEE Trans. Automat. Control, 47(1):53-69, 2002.

21. J. Wood. Modules and behaviours in $n \mathrm{D}$ systems theory. Multidimens. Systems Signal Process., 11(1-2):11-48, 2000.

22. J. Wood, E. Rogers, and D. H. Owens. A formal theory of matrix primeness. Math. Control Signals Syst., 11(1):40-78, 1998.

23. E. Zerz. Primeness of multivariate polynomial matrices. Systems Control Lett., 29(3):139_ 145, 1996.

24. E. Zerz and V. Lomadze. A constructive solution to interconnection and decomposition problems with multidimensional behaviors. SIAM J. Control Optim., 40(4):1072-1086, 2001/02. 